 for neatly organized compositions
}

Eline Van Geert \& Johan Wagemans Laboratory of Experimental Psychology, KU Leuven, Belgium

\section{Starting point}

\section{Order, complexity, and neatly organized compositions}

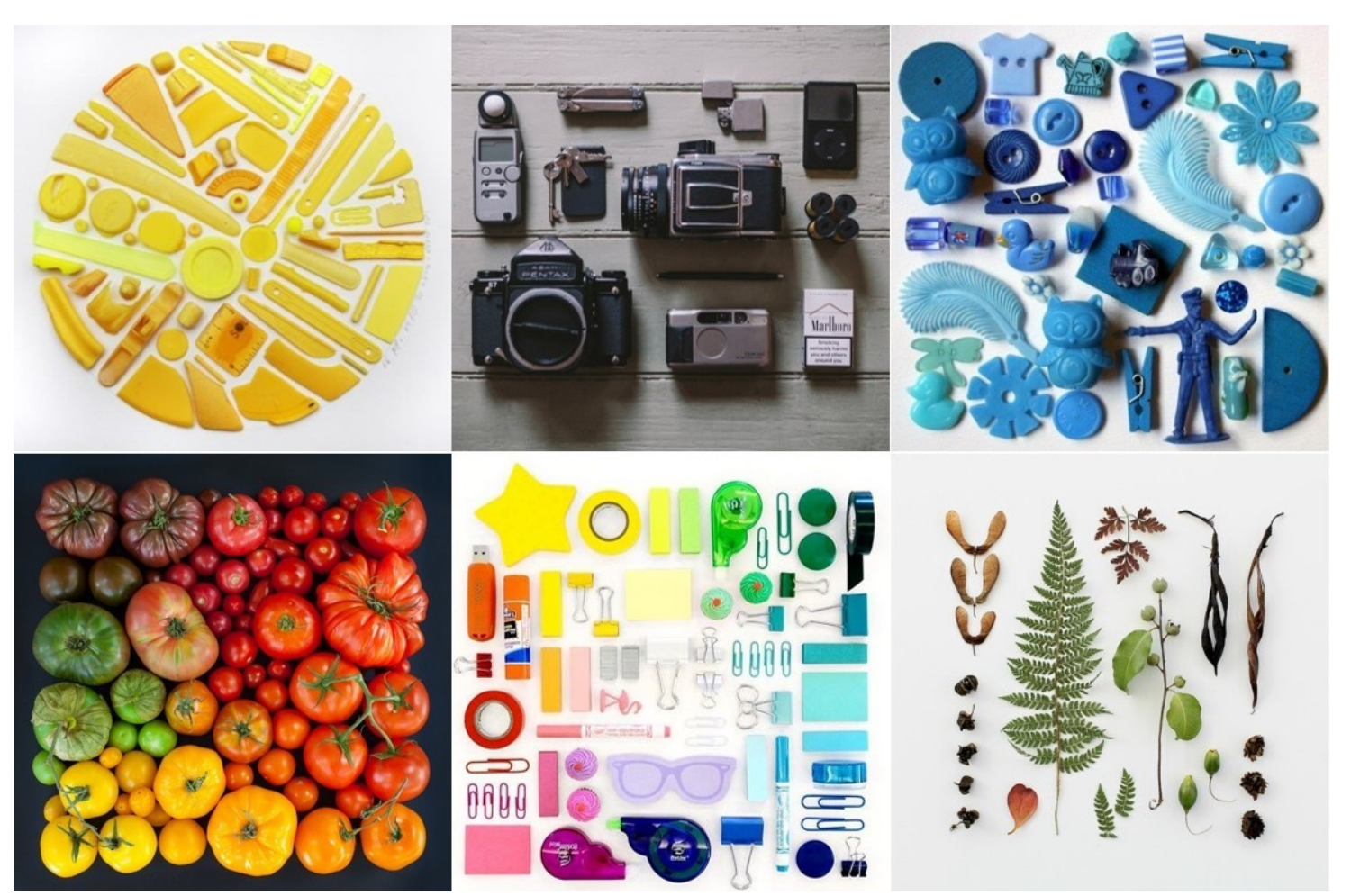

Images of a set of objects (or parts of objects) organized in an orderly way

Popular online (e.g., blog Things Organized Neatly ${ }^{\odot}$ )

Main question: Which factors are associated with aesthetic preferences for specific neatly organized compositions?

Complexity: aspects related to the quantity and variety of information in a stimulus Order: aspects related to the structure and organization in a stimulus

Relation order and complexity: antagonistic and complementary (Arnheim, 1966)

\section{Original study}
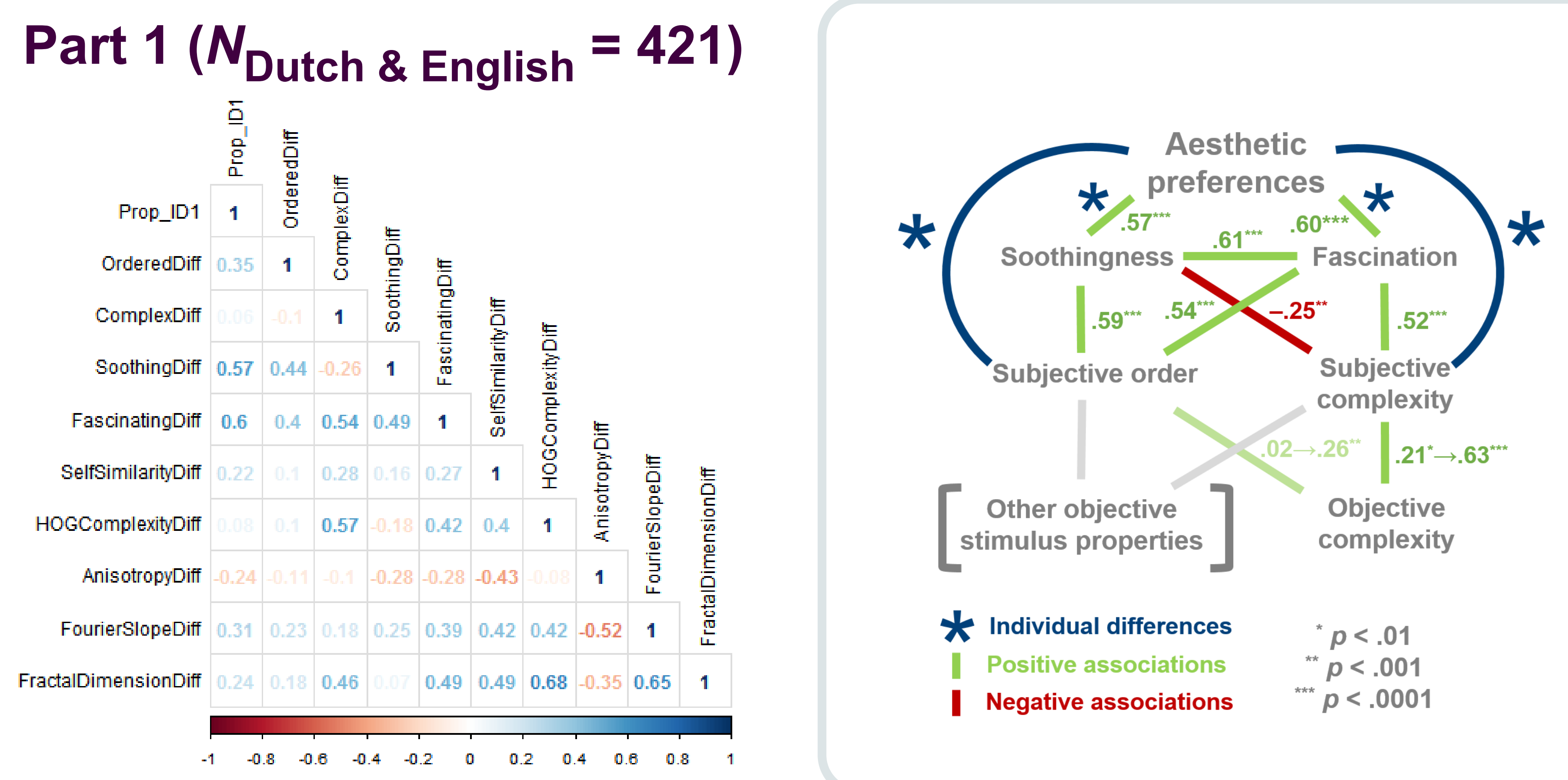

Part $2(N=84)$
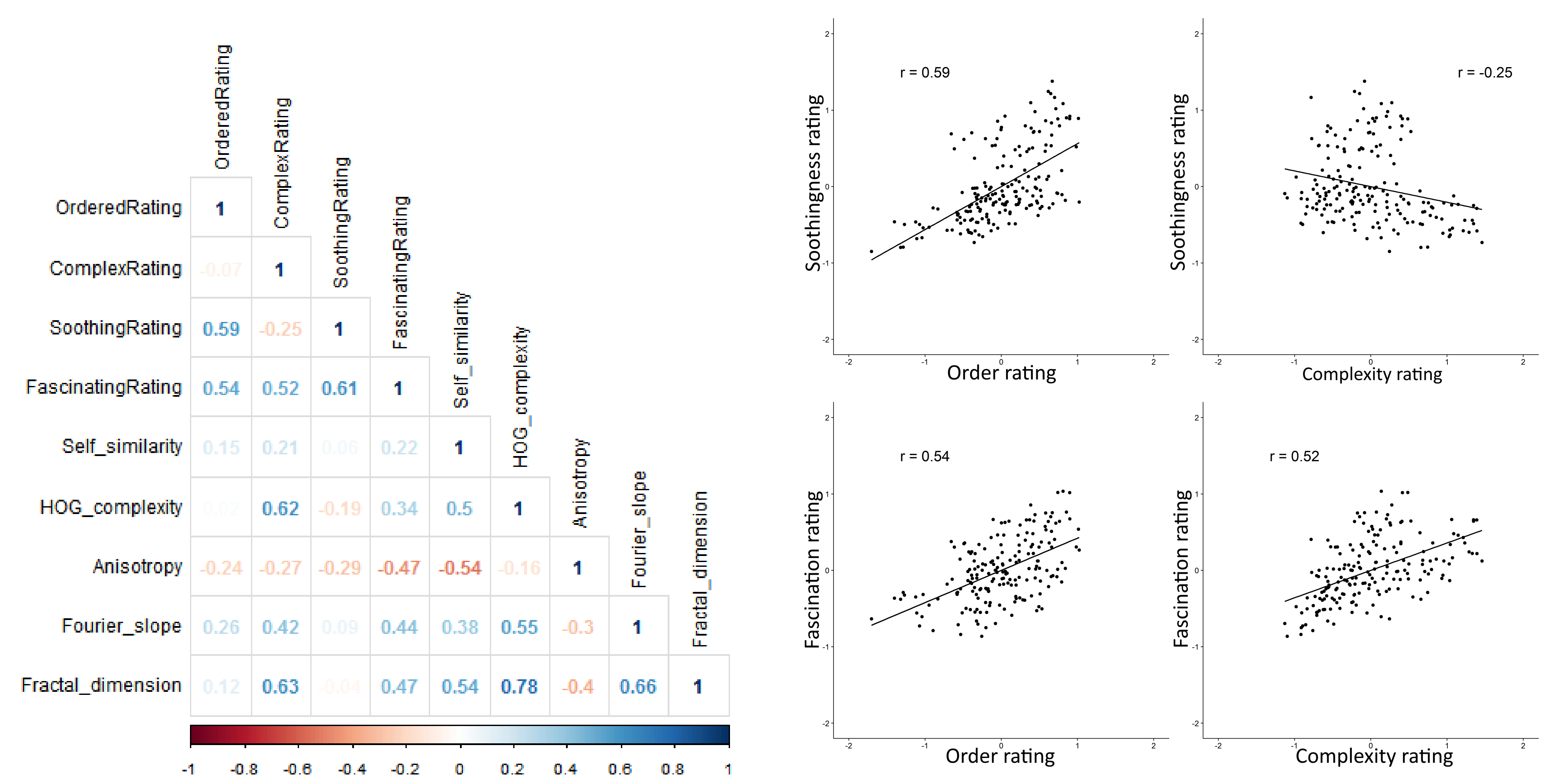

General associations:

- different types of aesthetic appreciation show positive associations (preferences, soothingness, and fascination)

- however, soothingness and fascination relate differently to order and complexity

$\rightarrow$ different pathways to aesthetic appreciation (Graf \& Landwehr, 2017)?

- rather combination of order and complexity than interaction which relates to aesthetic appreciation

Individual differences:

- individual differences in preference for order or complexity relate to scores on Personal Need for Structure (PNS), Openness, and age

\section{Procedure}

Part 1: Preferences (100 pairs) and personality

"Please click on the image you prefer"
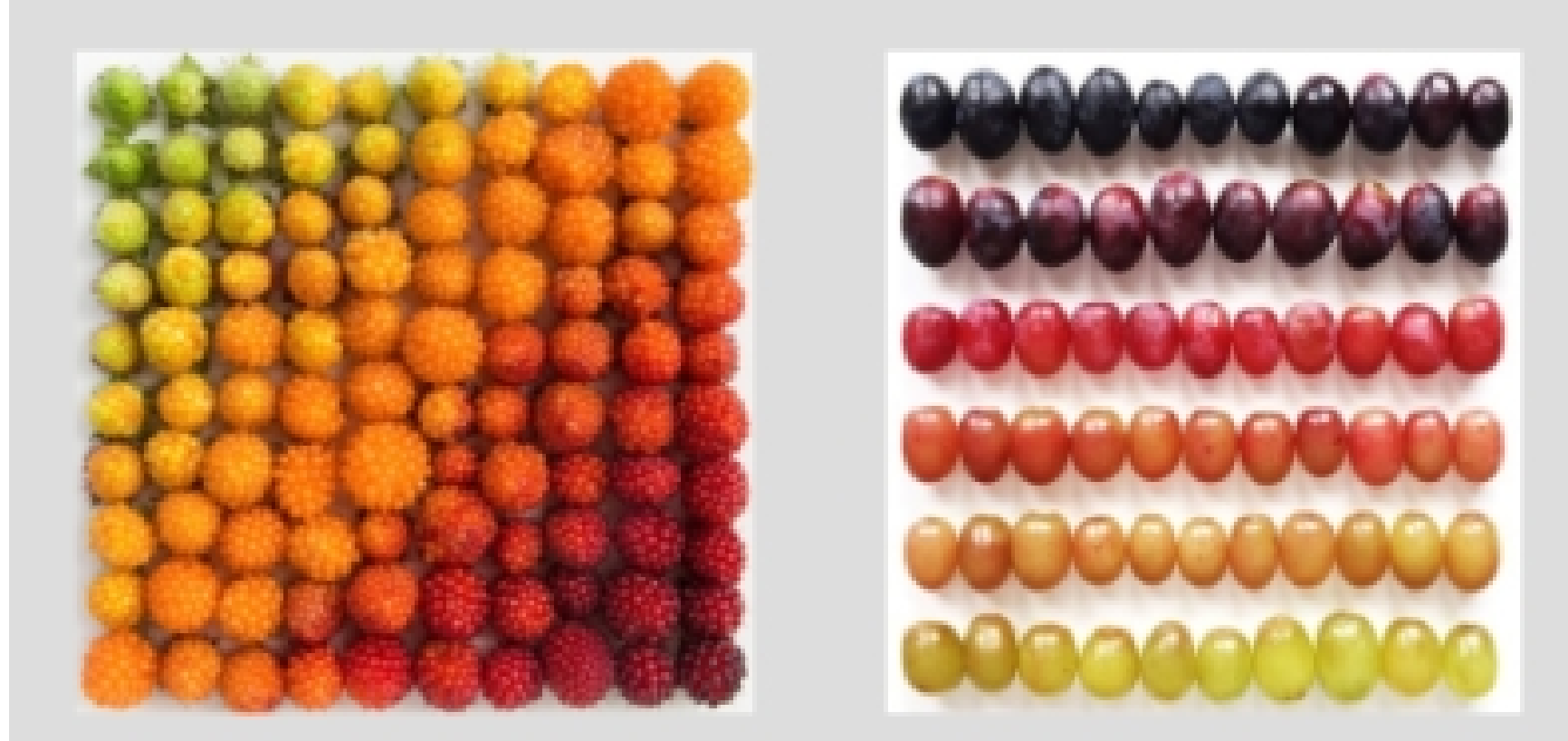

Personality questionnaires

- Big Five Inventory (BFI)

- Personal Need for Structure (PNS)

\section{Cross-cultural comparison}

Part $1\left(N_{\text {Native Dutch }}=356, N_{\text {Native Chinese }}=220\right)$
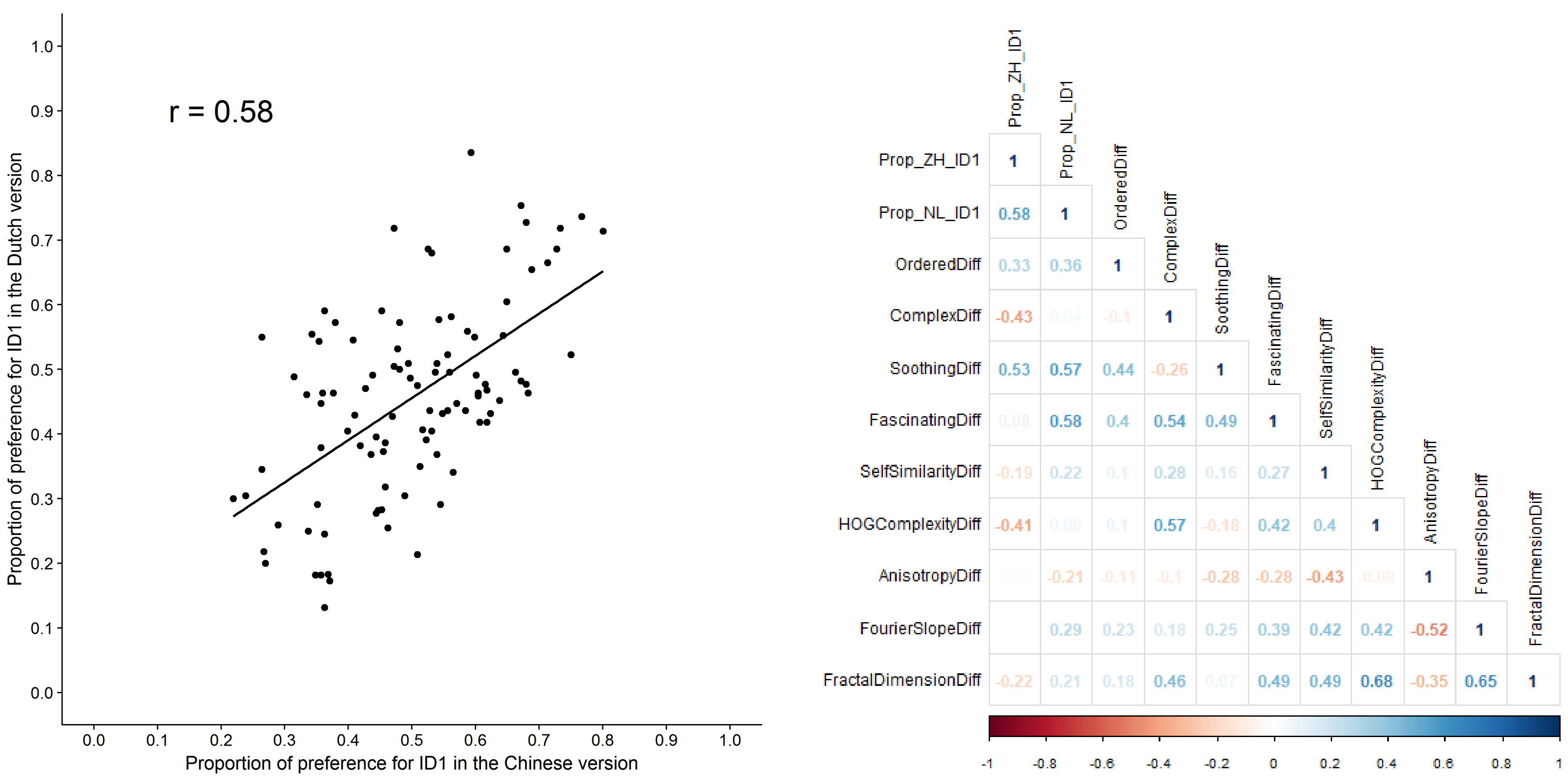

ZH

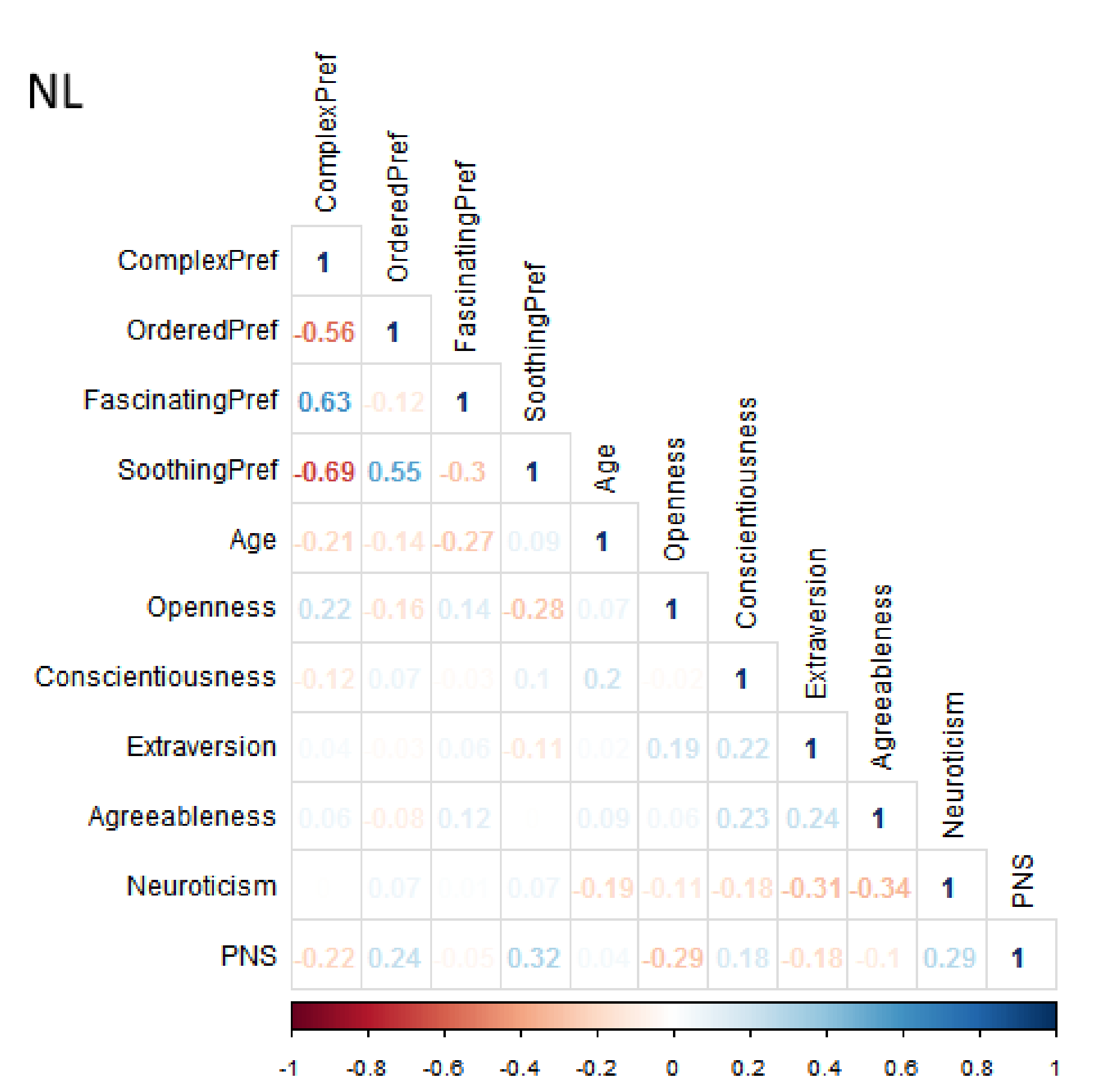

General associations:

- in both cultures, preferences relates to soothingness and order

- however, in the Chinese-speaking sample there is a preference for simplicity

- however, in the Dutch-speaking sample there is a preference for fascination

Individual differences:

- relation of Openness and age with preferences is consistent across cultures - relation of Personal Need for Structure (PNS) with preferences is the other way around in the Chinese-speaking sample compared to the Dutch-speaking one $\rightarrow$ is this due to a validity problem in the Chinese version of the questionnaire or is the difference in relation interpretable?

\section{References}

Arnheim, R. (1966). Order and complexity in landscape design. In R. Arnheim (Ed.), Toward a psychology of art: Collected essays (pp. 123-135). Berkeley and Los Angeles, CA: University of California Press. Graf, L. K. M., \& Landwehr, J. R. (2017). Aesthetic pleasure versus aesthetic interest: The two routes to aesthetic liking. Frontiers in Psychology, 8, 15. doi:10.3389/fpsyg. 2017.00015 